\title{
Celebrating today, inspiring tomorrow
}

\author{
Computational and mathematical models are at the core of myriad research developments across different \\ domains. We pay tribute to the importance of computational science by providing a dedicated home among the \\ Nature portfolio for this inspiring field.
}

C omputational science has made a positive impact on our world, contributing to numerous advancements that have been hailed as milestones by the scientific community and substantially changing the way we do science. To name a few, in 2013, computational science took the Nobel stage when the chemistry award was given to three researchers for developing computational modeling techniques, with origins dating back to the 1970 s, that are used to understand complex chemical processes and interactions. In 2019, the first-ever photograph of a black hole was generated $^{1}$, a historic and awe-inspiring moment in science that was possible due to the use of various technologies, including supercomputers and new algorithms in computational imaging. Also in 2019, Google's quantum supremacy announcement ${ }^{2}$ - that their quantum computer can make a calculation much faster than the fastest supercomputers available - was considered a milestone for many, and while there has been debate about these claims, there is no doubt that quantum computing is advancing at a fast pace. In 2020, a deep learning model achieved incredibly high accuracy when predicting the three-dimensional structure of proteins - a breakthrough in biological research. The list goes on and on.

With a steady stream of accomplishments in its rear-view, the progress being made in computational science is accelerating like never before. Accordingly, now is the ideal moment to launch Nature Computational Science as a premier home for research in this exciting field, which promises to continue to enlighten, surprise and inspire us.
Our main goal is to promote and celebrate great advances in the field and to foster discussion of topics relevant to the community by bringing all computational scientists and enthusiasts together. The journal will aspire to be a place to publish multidisciplinary research, including not only the development of new computational methods that advance science, but also novel applications of existing approaches that tackle important challenges in our society.

In our first issue, we celebrate the diversity of topics in computational science. We have content that showcases advancements in computational materials science, including an Article on the prediction of materials properties using low-fidelity data by Chen et al., an Article on the design of high-entropy alloys by Singh et al., and a Comment by Horton et al. on the development of materials databases. We have computational biology Articles on evolutionary biology by Kuwahara and Gao, on cancer genomics by Yang et al. and on biophysics by Löhr et al., as well as a Brief Communication by Raucci et al. on an exciting new resource to make quantum chemistry more accessible to the community. This issue also features a Perspective by Liu et al. that advocates for the use of causality techniques from econometrics in computational science, and a Correspondence by Casari et al. on effectively recognizing contributions in open source ecosystems.

We also wanted to cover recent events that shaped 2020 and the impact they've had on the computational science community. In the wake of horrific police killings, the Black community called out racism in society and science, demanding racial justice. We virtually met and had an insightful chat with
Jenea Adams, a second-year PhD student who has created a platform to connect Black women of all intersections of identity in computational biology. We spoke to her about gender and race inequality, as well as about her successful initiative that has already inspired many young researchers in the field. We stand by our fellow Black academics, and we pledge to strive for diversity in our pieces and to make this journal a safe space to discuss issues related to systemic racism in science.

The year of 2020 was also marked by COVID-19, which has made a monumental impact on our lives and society as a whole. It has also brought the computational science community together to address the enormous challenge of reducing the spread of the virus and developing treatments and vaccines at an unprecedented speed. This issue includes content related to the ongoing pandemic, including a Review by Galindez et al. on computational drug repurposing studies and lessons learned for future outbreaks, as well as Comments on pandemic data curation by Kraemer et al. and on COVID-19 models by Eggo et al.

We hope our readers are as excited to dive into our content as we are to curate and highlight all of the exceptional work being done in computational science. Here's to a great new year, full of exciting achievements in the field that we look forward to being part of!

Published online: 14 January 2021 https://doi.org/10.1038/s43588-020-00021-8

\footnotetext{
References

1. Castelvecchi, D. Nature 568, 284-285 (2019).

2. Gibney, E. Nature 574, 461-462 (2019).
} 